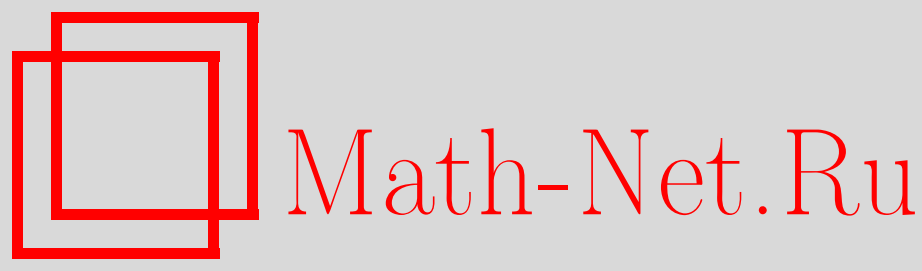

Г. В. Ефимов, Стационарное уравнение Шредингера нерелятивистской квантовой механики и функциональный интеграл, ТМФ, 2012, том 171, номер 3, 452-474

DOI: https://doi.org/10.4213/tmf6926

Использование Общероссийского математического портала Math-Net.Ru подразумевает, что вы прочитали и согласны с пользовательским соглашением http: //www . mathnet.ru/rus/agreement

Параметры загрузки :

IP: 54.162 .127 .20

26 апреля 2023 г., 12:24:16

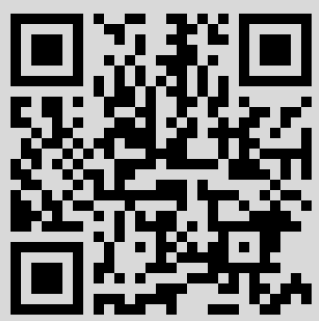




\title{
ФИЗИКА
}

Том 171, № 3

июнь, 2012

(C) 2012 г.

Г. В. Ефимов*

\section{СТАЦИОНАРНОЕ УРАВНЕНИЕ ШРЕДИНГЕРА НЕРЕЛЯТИВИСТСКОЙ КВАНТОВОЙ МЕХАНИКИ И ФУНКЦИОНАЛЬНЫЙ ИНТЕГРАЛ}

\begin{abstract}
Сформулирован метод представления решений однородных уравнений второго порядка в форме функционального интеграла, или интеграла по путям. В качестве примера получены решения уравнений второго порядка с постоянными коэффициентами и линейным потенциалом. Метод применен к нахождению общих решений стационарного уравнения Шредингера. Показано, как находятся спектр и собственные функции уравнения квантового осциллятора. Получено решение стационарного уравнения Шредингера в квазиклассическом приближении, не имеющее особенностей в точке поворота. В этом приближении найден коэффициент прохождения сквозь потенциальный барьер. Получено представление амплитуды упругого потенциального рассеяния в форме функционального интеграла.
\end{abstract}

Ключевые слова: однородные уравнения второго порядка, функциональный интеграл, стационарное уравнение Шредингера, квазиклассическое приближение, амплитуда упругого потенциального рассеяния.

\section{1. ВВЕДЕНИЕ}

Первоначально метод функционального интегрирования был предложен Фейнманом [1] как представление для функции Грина нестационарного уравнения Шредингера в нерелятивистской квантовой механике. Метод оказался весьма эффективным и в дальнейшем получил широкое распространение при решении разного рода физических задач. K настоящему времени имеется обширная литература (см., например, монографии [2]-[5]), в которой обсуждаются многочисленные аспекты метода и предлагаются различные способы вычисления возникающих функциональных интегралов.

Отметим, что этот метод обычно применяется только при решении нестационарных задач типа уравнения теплопроводности или нестационарного уравнения Шредингера. В квантовой механике это означает, что мы имеем дело с системами с неопределенной энергией. В то же время имеется обширный круг стационарных

* Объединенный институт ядерных исследований, Дубна, Россия.

E-mail: efimovg@theor.jinr.ru 
проблем, таких как нахождение спектра стационарных состояний, задача квантового рассеяния или прохождения сквозь потенциальный барьер, когда энергия точно задана, что требует решения стационарного уравнения Шредингера. Для таких задач, т. е. для решений стационарных уравнений с заданной энергией, решения в форме функционального интеграла еще не представлены, и мы хотим заполнить этот пробел.

Следует подчеркнуть, что функциональные интегралы хорошо определены только по гауссовой мере, которая естественно возникает при решении линейных уравнений второго порядка. Знание общих решений стационарных уравнений второго порядка даст возможность дальнейшего более глубокого изучения многочисленных задач физики.

В качестве примера мы получили решения уравнений второго порядка с постоянными коэффициентами и линейным потенциалом, а также нашли спектр и волновые функции квантового осциллятора. Основной результат состоит в том, что получены волновые функции в квазиклассическом приближении, которые справедливы в окрестности точек поворота, где обычное приближение не имеет места, и вычислена вероятность туннелирования сквозь потенциальный барьер. Кроме того, получено представление амплитуды упругого рассеяния на короткодействующем потенциале.

\section{2. ПРЕДВАРИТЕЛЬНЫЕ ЗАМЕЧАНИЯ}

Обычный подход к изучению функционального интеграла, или интеграла по путям, исходит из первоначальной идеи Фейнмана. А именно, напишем объект типа

$$
I=\int D \Phi e^{i S[\phi]}
$$

где $\Phi$ - некоторое поле, а функционал $S[\Phi]$ является действием. Затем исследуем написанное выражение, чтобы придать ему математический смысл и найти способы, как проводить конкретные вычисления. При таком подходе выражение (1) имеет самостоятельный смысл и не совсем ясно, имеет ли написанное выражение какое-либо отношение к какому-то уравнению. Необходимы дополнительные усилия, чтобы показать, что постулированный интеграл удовлетворяет какому-то определенному уравнению. В частности, в пионерской работе Фейнмана это было нестационарное уравнение Шредингера. Подобный подход традиционно проводится практически во всех многочисленных последующих работах (в качестве примера можно сослаться на недавнюю статью Виттена [6]).

Однако возможна и другая точка зрения, заключающаяся в том, что метод функционального интегрирования просто является эффективным методом представления решений линейных дифференциальных уравнений второго порядка. Поэтому надо непосредственно исходить из конкретного уравнения с конкретной постановкой начальных и краевых условий и искать способ, как решение этой конкретной задачи может быть представлено в форме функционального интеграла.

Продемонстрируем схему наших рассуждений на типичном примере. Пусть задан оператор вида

$$
L_{x}=-\frac{1}{2} \frac{d^{2}}{d x^{2}}+W(x)
$$


ТАБЛИЦА 1

\begin{tabular}{|c|c|c|}
\hline & Уравнение & Формальное решение \\
\hline 1 & $i \frac{d}{d t} \Psi=L_{x} \Psi$, & $\Psi=e^{-i t L_{x}} \Psi_{0}=\mathbf{U}_{t} \Psi_{0}$ \\
2 & $L_{x} \Psi=J$, & $\Psi=\frac{1}{L_{x}} J=\int_{0}^{\infty} d v e^{-v L} J=\int_{0}^{\infty} d v \mathbf{U}_{-i v} J$ \\
3 & $L_{x} \Psi=0$, & $\Psi=\delta\left(L_{x}\right) u_{0}=\int_{-\infty}^{\infty} \frac{d t}{2 \pi} e^{-i t L_{x}} u_{0}=\int_{-\infty}^{\infty} \frac{d t}{2 \pi} \mathbf{U}_{t} u_{0}$ \\
\hline
\end{tabular}

Типичные уравнения, которые приходится решать в разных ситуациях, и их формальные решения приведены в табл. 1

В этих формальных решениях присутствует дифференциальный оператор

$$
\mathbf{U}_{t}=e^{-i t L_{x}} \rightarrow \mathbf{U}_{z}=e^{-i z L_{x}},
$$

где параметр $t$ вполне может быть как вещественной, так и комплексной величиной $z=t+i v$. Этот оператор действует на некоторую заданную функцию, так что задача состоит в определении действия этого оператора $\mathbf{U}_{z}$ на функции от $x$

$$
U_{z}(x)=\mathbf{U}_{z} u_{0}(x),
$$

где $u_{0}(x)$ - некоторая достаточно гладкая функция. Функция $U_{z}(x)$ имеет определенные аналитические свойства в комплексной плоскости $z=t+i v$. Поэтому могут быть определены интегралы вида

$$
\int_{\Gamma} d z U_{z}(x),
$$

где $\Gamma$ - некоторый контур в комплексной плоскости $z$, проведенный в области аналитичности функции $U_{z}(x)$.

2.1. Оператор $\mathbf{U}_{z}$ и функциональный интеграл. Рассмотрим оператор $\mathbf{U}_{z}$. Далее мы будем использовать технику вычислений, изложенную в монографиях [7], [8]. Начнем с вещественных $z=t$. Имеется два оператора в показателе экспоненты: $d^{2} / d x^{2}$ и $W(x)$, которые не коммутируют между собой. Для того чтобы с ними можно было обращаться как с коммутирующими операторами, вводится так называемое Т-произведение

$$
\begin{aligned}
\mathbf{U}_{t} & =e^{-i t L_{x}}=\exp \left\{-i t\left[-\frac{1}{2} \frac{d^{2}}{d x^{2}}+W(x)\right]\right\}= \\
& =\mathrm{T}\left\{\exp \left[\frac{i}{2} \int_{0}^{t} d \tau \frac{d^{2}}{d x_{\tau}^{2}}\right] \exp \left[-i \int_{0}^{t} d \tau W\left(x_{\tau}\right)\right]\right\},
\end{aligned}
$$

под знаком которого операторы можно считать коммутирующими. Цена этой коммутации состоит в том, что операторы $d / d x_{\tau}$ и $x_{\tau}$ приобретают упорядочивающий 
индекс $\tau$, т. е. переменную, от которой они, по существу, не зависят и которая лишь определяет порядок расстановки некоммутирующих операторов.

Следующий шаг состоит в том, чтобы в формуле (4) квадратичную форму по $d / d x_{\tau}$ превратить в линейную. Для этого используется интегральное представление

$$
\begin{gathered}
\exp \left[\frac{i}{2} \int_{0}^{t} d \tau \frac{d^{2}}{d x_{\tau}^{2}}\right]=\int \frac{D \nu}{C} \exp \left[\frac{i}{2} \int_{0}^{t} d \tau \nu^{2}(\tau)+\int_{0}^{t} d \tau \nu(\tau) \frac{d}{d x_{\tau}}\right] \\
\int \frac{D \nu}{C} \exp \left[\frac{i}{2} \int_{0}^{t} d \tau \nu^{2}(\tau)\right]=1
\end{gathered}
$$

Так возникает гауссов функциональный интеграл. Подчеркнем, что нормировочная константа $C$, по существу, является бесконечной величиной, так что само представление (5) имеет смысл несобственного интеграла.

Пусть $u(x)$ - некоторая произвольная функция. Тогда

$\mathbf{U}_{t} u(x)=\int \frac{D \nu}{C} \exp \left[\frac{i}{2} \int_{0}^{t} d \tau \nu^{2}(\tau)+\int_{0}^{t} d \tau W\left(x_{\tau}+\int_{\tau}^{t} d \tau^{\prime} \nu\left(\tau^{\prime}\right)\right)\right] u\left(x+\int_{0}^{t} d \tau^{\prime} \nu\left(\tau^{\prime}\right)\right)$.

Здесь мы воспользовались представлением (5) и выполнили операцию сдвига под знаком Т-произведения.

Функции $W(x)$ и $u(x)$ должны быть аналитичны в окрестности вещественной оси по переменной $x$ так, чтобы на них была определена операция сдвига

$$
e^{a d / d x} W(x)=W(x+a), \quad e^{a d / d x} u(x)=u(x+a) .
$$

Этому требованию не удовлетворяют кусочно непрерывные потенциалы типа прямоугольной ямы.

Выделим из интегрирования по всем функциям $\nu(\tau)$ постоянную составляющую $\nu(\tau)=\frac{1}{t} \int_{0}^{t} d \tau \nu(\tau)+\frac{d}{d \tau}\left[\int_{0}^{\tau} d \tau^{\prime} \nu\left(\tau^{\prime}\right)-\frac{\tau}{t} \int_{0}^{t} d \tau^{\prime} \nu\left(\tau^{\prime}\right)\right]=\frac{y}{t}+\dot{\xi}(\tau), \quad \xi(0)=\xi(t)=0$.

Тогда получим

$$
\begin{aligned}
\mathbf{U}_{t} u(x)= & \int_{-\infty}^{\infty} \frac{d y}{C_{0}} \int \frac{D \xi}{C} \exp \left\{i \frac{y^{2}}{2 t}+\frac{i}{2} \int_{0}^{t} d \tau \dot{\xi}^{2}(\tau)+\right. \\
& \left.+i \int_{0}^{t} d \tau W\left[x+\left(1-\frac{\tau}{t}\right) y-\xi(\tau)\right]\right\} u(x+y)= \\
= & \int_{-\infty}^{\infty} \frac{d y}{\sqrt{2 \pi i t}} e^{i y^{2} / 2 t} H_{t}(x, y) u(x+y),
\end{aligned}
$$

где константа $C_{0}=\sqrt{2 \pi i t}$ определена из условия нормировки и

$$
\begin{gathered}
H_{t}(x, y)=\int \frac{D \xi}{C} \exp \left\{\frac{i}{2} \int_{0}^{t} d \tau \dot{\xi}^{2}(\tau)+i \int_{0}^{t} d \tau W\left[x+\left(1-\frac{\tau}{t}\right) y-\xi(\tau)\right]\right\} \\
\xi(0)=\xi(t)=0 .
\end{gathered}
$$

Таким образом, мы получили результат действия оператора на произвольную функцию в форме функционального интеграла, или интеграла по путям, как сейчас принято говорить. 
Переход в представлении (7) к комплексной переменной $t \rightarrow z=t+i v$ понимается как аналитическое продолжение функции $H_{t}(x, y)$ по $t$.

Представления (7), (8) позволяют математически определить приведенные в табл. 1 формальные выражения и, таким образом, найти решения исходных уравнений.

2.2. Однородное уравнение. Будем рассматривать однородные уравнения вида

$$
L_{x} u(x)=0,
$$

где оператор $L_{x}$ имеет вид (2) с некоторой заданной функцией $W(x)$. Задача состоит в том, чтобы найти два независимых решения однородного уравнения (9).

Введем функцию

$$
U_{z}(x)=\mathbf{U}_{z} u_{0}(x)=e^{-i z L_{x}} u_{0}(x), \quad z=t+i v \in \mathbb{C} .
$$

Здесь функция $u_{0}(x)$ произвольна, от нее требуется, чтобы на ней был определен оператор $L_{x}$ и чтобы она содержала весь импульсный спектр. В качестве простейшей такой функции можно выбрать $u_{0}(x)=\delta\left(x-x_{0}\right)$, или $u_{0}(x)=\delta(x)+x_{0} \delta^{\prime}(x)$, где функция $\delta(x)$ четная, а функция $\delta^{\prime}(x)$ нечетная.

Будем далее рассматривать функцию

$$
U_{z}(x)=\mathbf{U}_{z} \delta(x)=e^{-i z L_{x}} \delta(x), \quad z=t+i v \in \mathbb{C} .
$$

Задача состоит в том, чтобы определить поведение функции $U_{z}(x)$ во всей комплексной плоскости комплексного переменного $z=t+i v \in \mathbb{C}$, найти все ее особенности, а также области роста и убывания. Функция $U_{z}(x)$ удовлетворяет уравнению

$$
L_{x} U_{z}(x)=i \frac{d}{d z} U_{z}(x)
$$

Выберем некоторый контур $Г$ в комплексной плоскости $\mathbb{C}$ и рассмотрим функцию

$$
u(x)=\int_{\Gamma} d z U_{z}(x)
$$

Эта функция удовлетворяет однородному уравнению

$$
L_{x} u(x)=i \int_{\Gamma} d z \frac{d}{d z} U_{z}(x)=i\left[U_{z_{+}}(x)-U_{z_{-}}(x)\right]=0,
$$

если на концах контура интегрирования значения функции совпадают, $U_{z_{+}}(x)=$ $U_{z_{-}}(x)$, в частности $U_{z_{+}}(x)=U_{z_{-}}(x)=0$.

Таким образом, задача сводится к определению аналитических свойств функции $U_{z}(x)$ в комплексной плоскости $z \in \mathbb{C}$ и поиску двух независимых контуров $\Gamma$, на концах которых функция $U_{z}(x)$ принимает равные значения, в частности равные нулю. Для этой цели мы применим метод функционального интегрирования, или метод интегрирования по путям в функциональном пространстве. Мы будем рассматривать стационарное уравнение Шредингера, хотя предлагаемый метод применим и к другим уравнениям вида (9). 
ПримеР 1. В качестве простого примера покажем, как известные уравнения второго порядка могут быть решены предлагаемым способом. Рассмотрим два уравнения второго порядка с постоянными коэффициентами

$$
\left(-\frac{d^{2}}{d x^{2}}-\omega^{2}\right) u(x)=0, \quad\left(-\frac{d^{2}}{d x^{2}}+\kappa^{2}\right) v(x)=0,
$$

общие решения которых записываются как

$$
u(x)=a e^{i \omega x}+b e^{-i \omega x}, \quad v(x)=a e^{\kappa x}+b e^{-\kappa x} .
$$

Решим эти уравнения предложенным выше способом. Имеем

$$
\begin{aligned}
U_{z} & =\exp \left[-\frac{i z}{2}\left(-\frac{d^{2}}{d x^{2}}-\omega^{2}\right)\right] \delta(x)=\int \frac{d p}{2 \pi} \exp \left(i p x-\frac{i}{2} z p^{2}+\frac{i}{2} z \omega^{2}\right)= \\
& =\frac{1}{\sqrt{-i 2 \pi z}} \exp \left(\frac{i}{2} z \omega^{2}+i \frac{x^{2}}{2 z}\right), \\
V_{z} & =\exp \left[-\frac{i z}{2}\left(-\frac{d^{2}}{d x^{2}}+\kappa^{2}\right)\right] \delta(x)=\int \frac{d p}{2 \pi} \exp \left(i p x-i z p^{2}-\frac{i}{2} z \kappa^{2}\right)= \\
& =\frac{1}{\sqrt{-i 2 \pi z}} \exp \left(-\frac{i}{2} z \kappa^{2}+\frac{i}{2} \frac{x^{2}}{4 z}\right) .
\end{aligned}
$$

В комплексной плоскости $z=t+i v=r e^{i \phi}$ получим для модулей этих функций

$$
\left|U_{z}\right|=\frac{1}{\sqrt{2 \pi r}} \exp \left[\frac{1}{2}\left(-\omega^{2} r+\frac{x^{2}}{r}\right) \sin \phi\right], \quad\left|V_{z}\right|=\frac{1}{\sqrt{2 \pi r}} \exp \left[\frac{1}{2}\left(\kappa^{2} r+\frac{x^{2}}{r}\right) \sin \phi\right] .
$$

Функция $U_{z}$ ограничена на вещественной оси при $x \neq 0$. В комплексной плоскости в точке $z=0$ она имеет существенную особенность, при больших $r$ убывает в верхней полуплоскости и растет в нижней, а при малых $r$ растет в верхней полуплоскости и убывает в нижней. Поэтому в качестве контуров интегрирования выберем $\Gamma_{1}=$ $[0, \infty]$ и $\Gamma_{2}=[-\infty, 0]$. В результате получим

$$
\begin{aligned}
& u_{1}(x)=\int_{\Gamma_{1}} d z U_{z}=\int_{0}^{\infty} \frac{d z}{\sqrt{-i 2 \pi z}} \exp \left(\frac{i}{2} z \omega^{2}+i \frac{x^{2}}{2 z}\right)=c_{1} e^{i \omega x} \\
& u_{2}(x)=\int_{\Gamma_{2}} d z U_{z}=\int_{-\infty}^{0} \frac{d z}{\sqrt{-i 2 \pi z}} \exp \left(\frac{i}{2} z \omega^{2}+i \frac{x^{2}}{2 z}\right)=c_{2} e^{-i \omega x} .
\end{aligned}
$$

Функция $V_{z}$ ограничена на вещественной оси при $x \neq 0$. В комплексной плоскости в точке $z=0$ она имеет существенную особенность, а при больших и малых $r$ растет в верхней полуплоскости и убывает в нижней. Поэтому в качестве контуров интегрирования выберем $C_{1}=[-i \infty, 0]$ и $C_{2}=[i a-\infty, i a+\infty]$. В результате получим

$$
\begin{aligned}
& v_{1}(x)=\int_{C_{1}} d z V_{z}=\int_{-i \infty}^{0} \frac{d z}{\sqrt{-i 2 \pi z}} \exp \left(-\frac{i}{2} z \kappa^{2}+\frac{i}{2} \frac{x^{2}}{4 z}\right)=c_{1} e^{-\kappa x} \\
& v_{2}(x)=\int_{C_{2}} d z V_{z}=\int_{i a-\infty}^{i a+\infty} \frac{d z}{\sqrt{-i 2 \pi z}} \exp \left(-\frac{i}{2} z \kappa^{2}+\frac{i}{2} \frac{x^{2}}{4 z}\right)=c_{2} e^{\kappa x}
\end{aligned}
$$




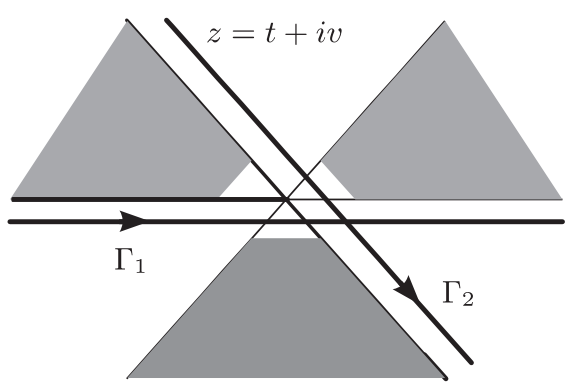

Рис. 1. Аналитические особенности функции $U_{z}(x)$ в комплексной плоскости $z=t+i v$. Имеется разрез в точке $z=0$. Области роста затемнены. $\Gamma_{1}$ и $\Gamma_{2}-$ два возможных контура интегрирования.

Пример 2. В качестве второго примера покажем, как решается уравнение с линейным потенциалом, которое в упрощенном виде записывается как

$$
\left(-\frac{d^{2}}{d x^{2}}+x\right) u(x)=0 .
$$

Для функции $U_{t}(x)$ получим

$$
\begin{aligned}
U_{t}(x) & =\exp \left[-i t\left(-\frac{d^{2}}{d x^{2}}+x\right)\right] \delta(x)=\int \frac{d p}{2 \pi} \exp \left[-i t\left(-\frac{d^{2}}{d x^{2}}+x\right)\right] e^{i p x}= \\
& =\frac{1}{\sqrt{4 i \pi(t-i 0)}} \exp \left(-i t x-i \frac{t^{3}}{12}+\frac{i}{2} t^{2}+\frac{i}{4} t\right) .
\end{aligned}
$$

Здесь мы воспользовались методом Т-упорядочивания (см., например, [8]):

$$
\begin{aligned}
\exp \left[-i t\left(-\frac{d^{2}}{d x^{2}}+x\right)\right] e^{i p x} & =\mathrm{T}\left\{\exp \left[i \int_{0}^{t} d \tau \frac{d^{2}}{d x_{\tau}^{2}}\right] \exp \left[-i \int_{0}^{t} d \tau x_{\tau}\right]\right\} e^{i p x}= \\
& =e^{-i t x} \exp \left[i \int_{0}^{t} d \tau\left(-i \tau+\frac{d}{d x}\right)^{2}\right] e^{i p x}= \\
& =\exp \left[-i(t-p) x-i \int_{0}^{t} d \tau(\tau-p)^{2}\right]= \\
& =\exp \left\{-i(t-p) x-\frac{i}{3}\left[(t-p)^{3}+p^{3}\right]\right\} .
\end{aligned}
$$

Аналитические особенности функции $U_{z}(x)$ при переходе к комплексным $t \rightarrow z=$ $t+i v$ показаны на рис. 1. Для двух независимых решений уравнения (14) имеем два контура $\Gamma_{1}$ и $\Gamma_{2}$.

Решение, определяемое контуром $\Gamma_{1}$, имеет вид

$$
\begin{aligned}
u_{1}(x) & =\int_{\Gamma_{1}} d z U_{z}(x)=\int d t \int \frac{d p}{2 \pi} \exp \left\{-i(t-p) x-\frac{i}{3}\left[(\tau-p)^{3}+p^{3}\right]\right\}= \\
& =\int \frac{d t}{2 \pi} e^{-i t x-i t^{3} / 3} \int \frac{d p}{2 \pi} e^{-i p^{3} / 3}=C \int_{0}^{\infty} d t \cos \left(t x+\frac{t^{3}}{3}\right)
\end{aligned}
$$


и является решением, обычно приводимым в литературе (см., например, [9]).

Второе решение, определяемое контуром $\Gamma_{2}$, записывается как

$$
\begin{aligned}
u_{2}(x)= & \int_{\Gamma_{2}} d z U_{z}(x)=c\left[\int_{0}^{\infty} \frac{d v}{\sqrt{v}} \exp \left(-x v-\frac{v^{3}}{12}-\frac{i}{2} v^{2}+\frac{v}{4}\right)+\right. \\
& \left.+\int_{0}^{\infty} \frac{d t}{\sqrt{t}} \exp \left(-i x t-i \frac{t^{3}}{12}+\frac{i}{2} t^{2}+\frac{i}{4} t\right)\right]
\end{aligned}
$$

Эта функция растет при $x \rightarrow-\infty$.

\section{3. СТАЦИОНАРНОЕ УРАВНЕНИЕ ШРЕДИНГЕРА}

Будем решать стационарное уравнение Шредингера

$$
\left[-\frac{\hbar^{2}}{2 m} \frac{d^{2}}{d x^{2}}+V(x)\right] \Psi(x)=E \Psi(x) .
$$

Перепишем это уравнение в форме однородного уравнения

$$
L_{x} \Psi(x)=0,
$$

где

$$
L_{x}=H_{x}-E=\left[-\frac{\hbar^{2}}{2 m} \frac{d^{2}}{d x^{2}}-T_{E}(x)\right], \quad T_{E}(x)=E-V(x)
$$

Здесь величина $T_{E}(x)$ является кинетической энергией частицы в точке $x$.

Введем функцию

$$
U_{z}(x)=e^{-i z L_{x} / \hbar} \delta(x), \quad L_{x} U_{z}(x)=i \hbar \frac{d}{d z} U_{z}(x)
$$

В представлении (20) параметр $z$ будем рассматривать как комплексную переменную $z=t+i v$. Введем представление

$$
\Psi_{\Gamma}(x)=\int_{\Gamma} d z U_{z}(x)
$$

Согласно сказанному выше задача состоит в том, чтобы исследовать аналитические свойства функции $U_{z}(x)=U_{t+i v}(x)$ и найти два независимых контура $\Gamma_{1}$ и $\Gamma_{2}$, на концах которых функция $U_{z}(x)$ принимает равные значения или обращается в нуль. Тогда представление (21) определяет два независимых решения стационарного уравнения Шредингера.

В случае, когда потенциал $V(x)$ аналитичен в некоторой окрестности вещественной оси $x$, для $U_{z}(x)$ можно написать представление в форме функционального 
интеграла (см., например, [7], [8]):

$$
\begin{aligned}
U_{z}(x)= & e^{-i z L_{x} / \hbar} \delta(x)=\sqrt{\frac{m}{2 i \pi \hbar z}} \int_{\xi(0)=0, \xi(z)=x} \frac{D \xi}{C} \times \\
& \times \exp \left\{\frac{i}{\hbar} \int_{0}^{z} d \tau\left[\frac{m}{2} \dot{\xi}^{2}(\tau)+T_{E}(\xi(\tau))\right]\right\}= \\
= & \sqrt{\frac{m}{2 i \pi \hbar z}} \exp \left[\frac{i}{\hbar}\left(\frac{m x^{2}}{2 z}+E z\right)\right] \int_{\chi(0)=\chi(z)=0} \frac{D \chi}{C} \times \\
& \times \exp \left\{\frac{i}{\hbar} \int_{0}^{z} d \tau\left[\frac{m}{2} \dot{\chi}^{2}(\tau)-V\left(x \frac{\tau}{z}+\chi(\tau)\right)\right]\right\}= \\
= & \sqrt{\frac{m}{2 i \pi \hbar}} \frac{1}{\sqrt{z}} \exp \left[\frac{i}{\hbar}\left(\frac{m x^{2}}{2 z}+E z\right)\right] \int_{\eta(0)=\eta(1)=0} \frac{D \eta}{C} \times \\
& \times \exp \left\{\frac{i}{\hbar} \int_{0}^{1} d \tau\left[\frac{m}{2} \dot{\eta}^{2}(\tau)-z V(x \tau+\sqrt{z} \eta(\tau))\right]\right\} .
\end{aligned}
$$

Из этого представления следует, что функциональный интеграл определен при вещественных $z=t$. Аналитические свойства функции $U_{z}(x)$ определяются, во-первых, множителем $\exp \left\{(i / \hbar)\left(m x^{2} / 2 z+E z\right)\right\} / \sqrt{z}$, показывающим, что при $x^{2} \neq 0$ имеются существенная особенность и разрез в точке $z=0$, и, во-вторых, поведением потенциала $V(z)$ в комплексной плоскости $z$. В общем случае больше сказать ничего нельзя. Необходимо исследовать функцию $U_{z}(x)$ для каждого конкретного потенциала.

\section{4. КВАНТОВЫЙ ОСЦИЛЛЯТОР}

Рассмотрим уравнение для линейного осциллятора

$$
\left(-\frac{1}{2} \frac{d^{2}}{d x^{2}}+\frac{\omega^{2}}{2} x^{2}-E\right) u(x)=0 .
$$

Мы не будем приводить здесь все возможные решения при произвольных $E$, покажем только, как получить известный спектр и волновые функции финитного движения. Для функции $U_{z}(x)$ известно точное выражение (подробнее см. [1], [8]):

$$
\begin{aligned}
U_{z}(x) & =\exp \left[-i z\left(-\frac{1}{2} \frac{d^{2}}{d x^{2}}+\frac{\omega^{2}}{2} x^{2}-E\right)\right] \delta\left(x-x_{0}\right)= \\
& =e^{i z E} \sqrt{\frac{\omega}{2 \pi i \sin (\omega z)}} \exp \left\{i \omega\left[\left(\frac{x+x_{0}}{2}\right)^{2} \operatorname{tg}\left(\frac{\omega z}{2}\right)-\left(\frac{x-x_{0}}{2}\right)^{2} \operatorname{ctg}\left(\frac{\omega z}{2}\right)\right]\right\} .
\end{aligned}
$$

В комплексной плоскости $z$ эта функция имеет существенные особенности в точках $z_{n}= \pm \pi n / \omega, n=0,1,2, \ldots$. Вне вещественной оси при больших $z$ рост или убывание определяется множителем $e^{i z E}$ и зависит от знака $E$.

Выберем контур интегрирования $\Gamma=[0, \pi / \omega]$. На этом контуре при $z=t$ из представления (23), воспользовавшись производящими функциями для полиномов Лагерра и Эрмита (см., например, [10]), получим для четных и нечетных функций 
соответственно

$$
\begin{aligned}
U_{t}^{(1)}(x) & =\exp \left[-i z\left(-\frac{1}{2} \frac{d^{2}}{d x^{2}}+\frac{\omega^{2}}{2} x^{2}-E\right)\right] \delta(x)= \\
& =\exp \left[-\frac{\omega x^{2}}{2}+i\left(E-\frac{\omega}{2}\right) t\right] \sqrt{\frac{\omega}{\pi}} \sum_{n=0}^{\infty} \frac{(-1)^{n}}{4^{n} n !} e^{-i 2 n \omega t} H_{2 n}(\sqrt{\omega} x) \sim \\
& \sim e^{i(E-\omega / 2) t} F_{1}\left(e^{-i 2 \omega t}\right)
\end{aligned}
$$

и

$$
\begin{aligned}
U_{t}^{(2)}(x) & =\exp \left[-i z\left(-\frac{1}{2} \frac{d^{2}}{d x^{2}}+\frac{\omega^{2}}{2} x^{2}-E\right)\right] \delta^{\prime}(x)= \\
& =\exp \left[-\frac{\omega x^{2}}{2}+i\left(E-\frac{\omega}{2}\right) t\right] \sqrt{\frac{\omega}{\pi}} \sum_{n=0}^{\infty} \frac{(-1)^{n}}{4^{n} n !} e^{-i(2 n+1) \omega t} H_{2 n+1}(\sqrt{\omega} x) \sim \\
& \sim e^{i(E-\omega-\omega / 2) t} F_{2}\left(e^{-i 2 \omega t}\right) .
\end{aligned}
$$

Согласно сказанному выше необходимо, чтобы функция $U_{t}(x)$ принимала равные значения на концах интервала интегрирования $U_{0}(x)=U_{\pi / \omega}(x)$. Легко видеть, что функции $F_{1}\left(e^{-i 2 \omega t}\right)$ и $F_{2}\left(e^{-i 2 \omega t}\right)$ периодичны на этом интервале. Функция $U_{t}^{(1)}(x)$ периодична лишь при $E_{2 n}=\omega(2 n+1 / 2)$, а функция $U_{t}^{(2)}(x)$ периодична лишь при $E_{2 n+1}=\omega(2 n+1+1 / 2)$.

Эти формулы определяют спектр осциллятора

$$
E_{n}=\omega\left(n+\frac{1}{2}\right)
$$

Волновая функция для собственного значения $E_{n}$ описывается известным выражением

$$
\Psi_{n}(x)=\int_{0}^{\pi / \omega} d t U_{t}(x)=c e^{-\omega x^{2} / 2} H_{n}(\sqrt{\omega} x) .
$$

\section{5. КВАЗИКЛАССИЧЕСКОЕ ПРИБЛИЖЕНИЕ}

Как известно, в случае, когда квантовая длина волны частицы $\lambda=\hbar / k$ меньше характерных длин рассматриваемой конкретной задачи, свойства системы близки к классическим и можно использовать так называемое квазиклассическое приближение (см., например, [9]). Напомним стандартный подход к решению стационарного уравнения Шредингера (18). Будем искать решение в виде

$$
\Psi(x)=e^{i S(x) / \hbar} .
$$

Для функции $S(x)$ получим уравнение

$$
\frac{1}{2 m}\left(S^{\prime}(x)\right)^{2}-\frac{i \hbar}{2 m} S^{\prime \prime}(x)=2 m(E-V(x))=p^{2}(x) .
$$

Формально квазиклассическое приближение - это разложение решения по малому параметру $\hbar$. Надо заметить, что параметр $\hbar$ сам по себе не может быть ни малым, ни большим, поскольку он имеет размерность действия. Тем не менее мы можем 
построить такое разложение, но справедливость полученного разложения должна быть оценена дополнительно.

Итак, в уравнении (25) имеется малый параметр ћ. Наша идея состоит в том, чтобы получить решение этого уравнения в виде ряда по этому малому параметру. Однако этот малый параметр является множителем при старшей производной в уравнении, так что решение имеет особенность в точке $\hbar=0$ и, строго говоря, не может быть разложено в регулярный ряд по $\hbar$. Эта проблема существенна в окрестностях точек, где функция $p^{2}(x)$ обращается в нуль. Это так называемые точки поворота, где импульс частицы обращается в нуль. Вдали от этих точек, когда

$$
\left|\frac{\hbar S^{\prime \prime}(x)}{p^{2}(x)}\right| \ll 1
$$

разложение по $\hbar$ вполне применимо, но имеет смысл асимптотического разложения. Именно условие (26) является критерием применимости квазиклассического приближения.

Итак, представим функцию $S(x)$ в виде

$$
S(x)=S_{0}(x)+\hbar S_{1}(x)+O\left(\hbar^{2}\right)
$$

и подставим это разложение в уравнение (25). Приравнивая коэффициенты при одинаковых степенях $\hbar$ к нулю, получим для первых двух коэффициентов

$$
\begin{aligned}
\left(S_{0}^{\prime}(x)\right)^{2} & =2 m(E-V(x))=p^{2}(x), \\
S_{1}(x) & =\frac{i}{2} \ln S_{0}^{\prime}(x)=\frac{i}{2} \ln p(x) .
\end{aligned}
$$

Имеются две области: физическая область $E>V(x)$, где частица находится над барьером и имеет вещественный импульс, и нефизическая область $E<V(x)$, где частица находится ниже барьера и имеет мнимый импульс, что в классической физике абсолютно запрещено. Тогда квазиклассическое решение уравнения Шредингера представляется как

$$
\Psi(x)= \begin{cases}\frac{C_{+}}{\sqrt{p(x)}} \exp \left[ \pm \frac{i}{\hbar} \int^{x} d x^{\prime} p\left(x^{\prime}\right)+O\left(\hbar^{2}\right)\right], & E>V(x), \\ \frac{C_{-}}{\sqrt{|p(x)|}} \exp \left[ \pm \frac{1}{\hbar} \int^{x} d x^{\prime}\left|p\left(x^{\prime}\right)\right|+O\left(\hbar^{2}\right)\right], & E<V(x) .\end{cases}
$$

Иэ этих формул ясно видно, что в точках поворота, где $p(x)=0$, решение не определено, в то время как точное решение не должно иметь в этих точках никаких особенностей.

\section{6. ФУНКЦИОНАЛЬНЫЙ ИНТЕГРАЛ И КВАЗИКЛАССИЧЕСКОЕ ПРИБЛИЖЕНИЕ}

Рассмотрим, как выглядит квазиклассическое приближение в представлении волновой функции в форме функционального интеграла. Это приближение может быть получено из представлений (21) и (22). 
Предел малых $\hbar$ означает, что в представлении

$$
\Psi(x)=\int_{\Gamma} \frac{d z}{\sqrt{z}} e^{i E z / \hbar} \int_{\chi(0)=0, \chi(z)=x} \frac{D \chi}{C} \exp \left\{\frac{i}{\hbar} \int_{0}^{z} d \tau\left[\frac{m \dot{\chi}^{2}(\tau)}{2}-V(\chi(\tau))\right]\right\}
$$

величина $1 / \hbar$ велика, $1 / \hbar \gg 1$, и надо вычислить этот интеграл методом перевала как по функциональной переменной $\xi$, так и по комплексной переменной $z$.

Сначала рассмотрим функциональный интеграл по $\xi$. Положим

$$
\chi(\tau)=\xi(\tau)+\eta(\tau), \quad \xi(0)=0, \quad \xi(z)=x, \quad \eta(0)=\eta(z)=0 .
$$

Считая $|\eta(\tau)|$ малым, получим

$$
\begin{aligned}
S_{t}[\chi] & =\int_{0}^{z} d \tau\left[\frac{m}{2} \dot{\chi}^{2}(\tau)-V(\chi(\tau))\right]=S_{t}[\xi+\eta]= \\
& =\int_{0}^{z} d \tau\left[\frac{m}{2} \dot{\xi}^{2}(\tau)-V(\xi(\tau))\right]+\frac{1}{2} \int_{0}^{z} d \tau \eta(\tau)\left[-m \frac{d^{2}}{d \tau^{2}}-V^{\prime \prime}(\xi(\tau))\right] \eta(\tau)+O\left(\eta^{3}\right) .
\end{aligned}
$$

Линейное слагаемое по $\eta(\tau)$ отсутствует, поскольку $\xi(\tau)$ удовлетворяет классическому уравнению движения частицы в потенциале $V(\xi)$

$$
m \ddot{\xi}(\tau)+V^{\prime}(\xi(\tau))=0, \quad \xi(0)=0, \quad \xi(z)=x .
$$

Гауссов функциональный интеграл по $\eta(\tau)$ равен [11]

$$
\begin{aligned}
\mathcal{N}(z) & =\int \frac{D \eta}{C} \exp \left\{\frac{i}{2 \hbar} \int_{0}^{z} d \tau \eta(\tau)\left[-m \frac{d^{2}}{d \tau^{2}}-V^{\prime \prime}(\xi(\tau))\right] \eta(\tau)\right\}= \\
& =\sqrt{\frac{\operatorname{det}\left[-m \frac{d^{2}}{d \tau^{2}}\right]}{\operatorname{det}\left[-m \frac{d^{2}}{d \tau^{2}}-V^{\prime \prime}(\xi(\tau))\right]}}=\sqrt{\frac{z}{\phi(z)}} .
\end{aligned}
$$

Функция $\phi(z)$ является решением задачи Коши уравнения

$$
m \ddot{\phi}(\tau)+V^{\prime \prime}(\xi(\tau)) \phi(\tau)=0, \quad \phi(0)=0, \quad \dot{\phi}(0)=1 .
$$

Для ненормированной волновой функции получим представление

$$
\Psi(x)=\int_{\Gamma} \frac{d z e^{i[E z+\mathcal{S}(z)] / \hbar}}{\sqrt{\phi(z)}}=\int_{\Gamma} d z \exp \left\{\frac{i}{\hbar}[E z+\mathcal{S}(z)]-\frac{1}{2} \ln \phi(z)\right\},
$$

где $\mathcal{S}(z)$ - действие на стационарной траектории, а функция $\phi(\tau)$ является решением задачи Коши уравнения (30).

Вычислим показатель экспоненты $F(z)=E z+\mathcal{S}(z)$ на классической траектории. У классического уравнения (29) существует первый интеграл

$$
\frac{m}{2} \dot{\xi}^{2}(\tau)-E+V(\xi(\tau))=\frac{m}{2} K(z),
$$

где функция $K(z)$ определяется соотношениями

$$
\begin{gathered}
\dot{\xi}(\tau)=\sqrt{K(z)+W(\xi(\tau))}, \quad W(\tau)=\frac{2}{m} T(\tau)=\frac{2}{m}[E-V(\xi(\tau))], \\
\frac{d \xi}{\sqrt{K(z)+W(\xi)}}=d \tau, \quad \int_{0}^{\xi(\tau)} \frac{d \xi}{\sqrt{K(z)+W(\xi)}}=\tau,
\end{gathered}
$$


и формула

$$
\int_{0}^{x} \frac{d \xi}{\sqrt{K(z)+W(\xi)}}=z
$$

является интегральным уравнением на функцию $K(z)$ при фиксированных $x$ и $E$ как при вещественных $z=t$, так и при комплексных $z=t+i v$. В частности, имеем

$$
\int_{0}^{x} \frac{K^{\prime}(z) d \xi}{2 \sqrt{(K(z)+W(\xi))^{3}}}=-1
$$

В итоге на классической траектории показатель экспоненты равен

$$
F(z)=E z+\mathcal{S}(z)=\frac{m}{2} \int_{0}^{x} \frac{d \xi[K(z)+2 W(\xi)]}{\sqrt{K(z)+W(\xi)}} .
$$

Для волновой функции получим представление

$$
\Psi(x)=\int_{\Gamma} \frac{d z e^{i F(z) / \hbar}}{\sqrt{\phi(z)}}=\int_{\Gamma} \frac{d z}{\sqrt{\phi(z)}} \exp \left\{i \frac{m}{2 \hbar} \int_{0}^{x} \frac{d \xi[K(z)+2 W(\xi)]}{\sqrt{K(z)+W(\xi)}}\right\} .
$$

Следующий шаг - вычисление интеграла (35) по z методом перевала. Сначала рассмотрим ситуацию, когда координата $x$ далека от точки поворота, т. е. $E-$ $2 m V(x) \neq 0$. Тогда $\phi(x) \neq 0$ и главный вклад в интеграл дает функция $F(z)$.

Найдем перевальную точку. Производные классического действия по $z$ равны

$$
\frac{d}{d z} F(z)=\frac{d}{d z}[E z+\mathcal{S}(z)]=\frac{m}{2} \int_{0}^{x} \frac{d \xi K(z) K^{\prime}(z)}{2 \sqrt{(K(z)+W(\xi))^{3}}}=-\frac{m}{2} K(t)
$$

и

$$
F^{\prime \prime}(z)=\mathcal{S}^{\prime \prime}(z)=-\frac{m}{2} K^{\prime}(z), \quad F^{\prime \prime \prime}(z)=\mathcal{S}^{\prime \prime \prime}(z)=-\frac{m}{2} K^{\prime \prime}(z) .
$$

В точке перевала имеем

$$
F^{\prime}\left(z_{\mathrm{c}}\right)=-\frac{m}{2} K\left(z_{\mathrm{c}}\right)=0, \quad K\left(z_{\mathrm{c}}\right)=0,
$$

и точка перевала определяется интегралом

$$
\int_{0}^{x} \frac{d \xi}{\sqrt{W(\xi)}}=\int_{0}^{x} \frac{d \xi}{\sqrt{(2 / m)[E-V(\xi)]}}=z_{\mathrm{c}}
$$

Вычислим поведение функции $K(z)$ в окрестности перевальной точки. Запишем при $z \rightarrow z_{\mathrm{c}}$

$$
z_{\mathrm{c}}-z=\int_{0}^{x} d \xi\left[\frac{1}{\sqrt{W(\xi)}}-\frac{1}{\sqrt{K(z)+W(\xi)}}\right] \rightarrow-2 m \frac{\sqrt{K(z)}}{V^{\prime}(0)}
$$

где при $\xi \rightarrow 0$

$$
W(\xi)=-\frac{2}{m} V^{\prime}(0) \xi+O\left(\xi^{2}\right) .
$$


Из полученного соотношения следует

$$
K(z)=\left(\frac{V^{\prime}(0)}{4 m}\right)^{2}\left(z-z_{\mathrm{c}}\right)^{2}+O\left(\left(z-z_{\mathrm{c}}\right)^{3}\right), \quad z \rightarrow z_{\mathrm{c}} .
$$

Таким образом, для показателя экспоненты в окрестности точки перевала получим разложение

$$
F(z)=\int_{0}^{x} d \xi \sqrt{2 m(E-V(\xi))}+\frac{\left(V^{\prime}(0)\right)^{2}}{48 m}\left(z-z_{\mathrm{c}}\right)^{3}+O\left(\left(z-z_{\mathrm{c}}\right)^{4}\right) .
$$

Подчеркнем, что в полученном разложении отсутствует квадратичное по $z-z_{\text {c }}$ слагаемое. Это соответствует так называемым нулевым модам (см., например, [5]).

Итак, в квазиклассическом приближении интеграл (35) равен

$$
\begin{aligned}
\Psi(x) & =\int_{\Gamma} \frac{d z}{\sqrt{\phi(z)}} \exp \left\{\frac{i}{\hbar}[E z+\mathcal{S}(z)]\right\}= \\
& =\frac{1}{\sqrt{\phi\left(z_{\mathrm{c}}\right)}} \exp \left\{\frac{i}{\hbar} \int_{0}^{x} d \xi \sqrt{2 m(E-V(\xi))}\right\} \cdot \text { const }, \\
\text { const } & \sim \int_{-\infty}^{\infty} d z \exp \left[\frac{i}{6 \hbar} \mathcal{S}^{\prime \prime \prime}\left(z_{\mathrm{c}}\right)\left(z-z_{\mathrm{c}}\right)^{3}\right]
\end{aligned}
$$

Функция $\phi(z)$ является решением задачи Коши уравнения (30). Имеем цепочку равенств

$$
\begin{gathered}
m \ddot{\xi}(\tau)-T_{E}^{\prime}(\xi(\tau))=0, \\
{\left[m \frac{d^{2}}{d \tau^{2}}-T_{E}^{\prime \prime}(\xi(\tau))\right] \dot{\xi}(\tau)=0,} \\
m \frac{\dot{\xi}^{2}(\tau)}{2}-T_{E}(\xi(\tau))=\frac{m}{2} K(z), \\
\dot{\xi}(\tau)=\sqrt{K\left(z_{\mathrm{c}}\right)+W(\xi(\tau))}=\sqrt{W(\xi(\tau))},
\end{gathered}
$$

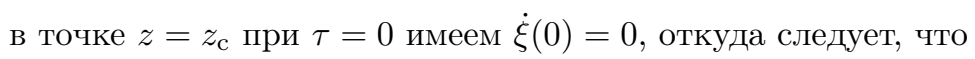

$$
\phi(\tau) \propto \dot{\xi}(\tau)
$$

и

$$
\phi\left(z_{\mathrm{c}}\right) \longrightarrow \dot{\xi}\left(z_{\mathrm{c}}\right)=\sqrt{W\left(\xi\left(z_{\mathrm{c}}\right)\right)}=\sqrt{W(x)} .
$$

Окончательно получаем известное выражение для ненормированной квазиклассической волновой функции

$$
\begin{aligned}
& \Psi_{-}(x)=\frac{1}{\left(p^{2}(x)\right)^{1 / 4}} \exp \left[ \pm \frac{i}{\hbar} \int^{x} d x^{\prime} p\left(x^{\prime}\right)\right], \\
& p^{2}(x)=2 m(E-V(x))=k^{2}-2 m V(x)>0 .
\end{aligned}
$$

Следует особо отметить, что данное представление справедливо только при $x$, далеких от точки поворота. 
6.1. Волновая функция в окрестности точки поворота. Стандартное представление квазиклассической волновой функции (27) не имеет места в окрестности точки поворота, где кинетическая энергия частицы равна нулю. Если же мы исходим из представления волновой функции в форме функционального интеграла и интеграла по соответствующему контуру в комплексной плоскости $z$ и будем вычислять этот интеграл методом перевала, то точка перевала не может быть в точке поворота, а будет как-то смещена в комплексную плоскость, так что интегрирование даст конечный результат для волновой функции в точке поворота.

Рассмотрим поведение волновой функции в окрестности точки поворота в квазиклассическом пределе, опираясь на представление $(31)$, где $\mathcal{S}(z)$ - действие на стационарной траектории, удовлетворяющей уравнению $(29)$, а функция $\phi(\tau)$ является решением задачи Коши уравнения (30).

Пусть $x_{1}=0$ - точка поворота. В окрестности этой точки при малых $\xi$ кинетическая энергия записывается как

$$
W(\xi)=\frac{2}{m}(E-V(\xi))=W_{1} \xi-W_{2} \frac{\xi^{2}}{2}+O\left(\xi^{3}\right), \quad W_{1}=-\frac{2}{m} V^{\prime}(0) .
$$

Будем считать, что $x>x_{1}=0$ и $V^{\prime}(0)<0$, т. е. рассматривается физическая область.

Функция $K(z)$ в этом пределе определяется интегралом

$$
\int_{0}^{x} \frac{d \xi}{\sqrt{K(z)+W_{1} \xi}}=z, \quad K(z)=\left(\frac{W_{1}}{4} z-\frac{x}{z}\right)^{2} .
$$

Уравнение для функции $\phi(\tau)$ в окрестности точки поворота приобретает вид

$$
\ddot{\phi}(\tau)+W_{2} \phi(\tau)=0, \quad \phi(0)=0, \quad \dot{\phi}(0)=1,
$$

откуда

$$
\phi(\tau)=\frac{\sin \left(\sqrt{W_{2}} \tau\right)}{\sqrt{W_{2}}}=\tau,
$$

и при малых аргументах можно считать, что $\phi(z)=z$. Согласно (32) имеем при $x \rightarrow 0$

$$
\dot{\xi}(\tau)=\sqrt{\left(\frac{W_{1}}{4} z-\frac{x}{z}\right)^{2}+W_{1} \xi(\tau)} \rightarrow \sqrt{\left(\frac{W_{1}}{4} z\right)^{2}+W_{1} \xi(\tau)} .
$$

Найдем перевальную точку. Производные классического действия по $z$ равны

$$
\frac{d}{d z}\left[\frac{i}{\hbar}(E z+\mathcal{S}(z))-\frac{1}{2} \ln \phi(z)\right]=-i \frac{m}{2 \hbar} K(z)-\frac{1}{2} \frac{\phi^{\prime}(z)}{\phi(z)}=0,
$$

получаем уравнение

$$
i \frac{m}{\hbar}\left(\frac{W_{1}}{4} z-\frac{x}{z}\right)^{2}+\frac{1}{z}=0
$$

откуда в пределе $x \rightarrow 0$ имеем

$$
z_{\mathrm{c}}=e^{-\pi i / 6}\left(\frac{16 \hbar}{m W_{1}^{2}}\right)^{1 / 3}=e^{-\pi i / 6}\left(\frac{4 m \hbar}{\left(V^{\prime}\left(x_{1}\right)\right)^{2}}\right)^{1 / 3} .
$$




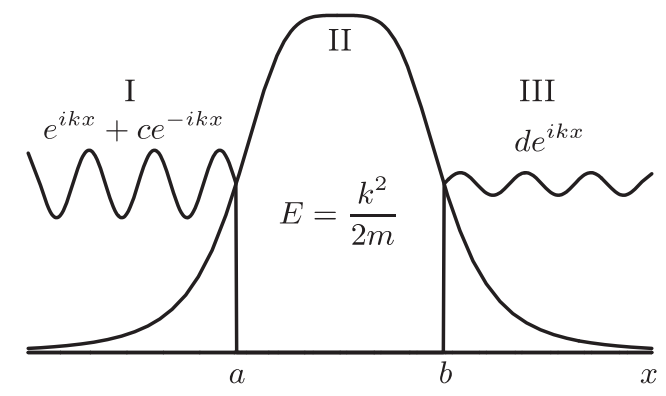

Рис. 2. Прохождение сквозь барьер.

В точке перевала

$$
K\left(z_{\mathrm{c}}\right)=\frac{2}{m} \kappa, \quad \kappa=e^{-\pi i / 6}\left(\frac{\left(V^{\prime}\left(x_{1}\right)\right)^{2} \hbar^{2}}{32 m}\right)^{1 / 3}
$$

и

$$
\dot{\xi}(x)=\sqrt{e^{-2 \pi i / 3} \kappa^{2}+p^{2}(x)},
$$

где $x_{1}=0$ - точка поворота. Таким образом, волновая функция конечна в точке поворота $\Psi(x) \sim 1 / \sqrt{\kappa}$.

При $x$, близких к $x_{1}$, можно пользоваться приближением

$$
\Psi_{-}(x)=\frac{C_{-}}{\left(e^{\mp \pi i / 3} \kappa^{2}+p^{2}(x)\right)^{1 / 4}} \exp \left[ \pm \frac{i}{\hbar} \int_{0}^{x} d x^{\prime} \sqrt{e^{\mp \pi i / 3} \kappa^{2}+p^{2}\left(x^{\prime}\right)}\right] .
$$

Если мы рассматриваем достаточно гладкие потенциалы, подобные показанному на рис. 2 , и считаем параметр $\hbar \ll 1$ малым, то в рамках нашего приближения можно предположить, что при нашей точности весьма разумным будет представление в физической области при $p^{2}(x)=k^{2}-2 m V(x)>0$

$$
\Psi_{-}(x)=\frac{C_{-}}{\left(e^{\mp \pi / 3} \kappa^{2}(x)+p^{2}(x)\right)^{1 / 4}} \exp \left[ \pm \frac{i}{\hbar} \int^{x} d x^{\prime} \sqrt{e^{\mp \pi / 3} \kappa^{2}\left(x^{\prime}\right)+p^{2}\left(x^{\prime}\right)}\right],
$$

а после аналогичных вычислений в нефизической области $p^{2}(x)=k^{2}-2 m V(x)<0$ имеем

$$
\Psi_{-}(x)=\frac{C_{-}}{\left(\kappa^{2}(x)+\left|p^{2}(x)\right|\right)^{1 / 4}} \exp \left[ \pm \frac{1}{\hbar} \int^{x} d x^{\prime} \sqrt{\kappa^{2}\left(x^{\prime}\right)+\left|p^{2}\left(x^{\prime}\right)\right|}\right],
$$

где функция $\kappa(x)$ дается равенством

$$
\kappa(x)=\left(\frac{m V^{\prime}(x) \hbar}{2}\right)^{1 / 3} .
$$

Покажем в заключение, как особенность $\hbar^{1 / 3}$ может быть непосредственно получена из уравнения (25). Пусть $\hbar$ мало и мы рассматриваем функцию $S(x)$ вблизи точки поворота $x \rightarrow x_{1}=0$. Положим

$$
S^{\prime}(x) \sim \hbar^{a}+\hbar^{b} x, \quad \hbar S^{\prime \prime}(x) \sim \hbar^{1+b}, \quad E-V(x) \sim x,
$$


где $a$ и $b$ - неопределенные коэффициенты. Тогда уравнение (25) приводит к соотношениям

$$
\hbar^{2 a}+\hbar^{a+b} x+\hbar^{1+b} \sim x
$$

откуда

$$
2 a=1+b, \quad a+b=0 \quad \Longrightarrow \quad a=\frac{1}{3} .
$$

Окончательно получаем

$$
S^{\prime}(0) \sim \hbar^{1 / 3}
$$

\section{7. ПРОХОЖДЕНИЕ СКВОЗЬ ПОТЕНЦИАЛЬНЫЙ БАРЬЕР}

В рамках предлагаемого представления рассмотрим квантовую задачу о прохождении частиц сквозь барьер. В квантовой механике возможно проникновение частиц сквозь потенциальный барьер в случае, когда энергия частицы меньше высоты барьера. Этой проблеме посвящено много исследований (см., например, [12]).

Задача ставится следующим образом. Рассматривается стационарная картина. Частица находится в поле потенциала, сосредоточенного в окрестности начала координат и убывающего достаточно быстро при $x \rightarrow \pm \infty$, как показано на рис. 2 . Имеется стационарный поток частиц, падающий слева на барьер. Частицы в основном отражаются от барьера, но частично проходят сквозь него. Надо найти соответствующие коэффициенты отражения и прохождения. Ищется решение стационарного уравнения Шредингера (18), удовлетворяющее асимптотическим граничным условиям

$$
\Psi(x) \longrightarrow \begin{cases}e^{i k x}+c(k) e^{-i k x}, & x \rightarrow-\infty \\ d(k) e^{i k x}, & x \rightarrow \infty .\end{cases}
$$

Необходимо вычислить коэффициенты $c(k)$ и $d(k)$, которые соответственно определяют вероятность отражения и прохождения сквозь потенциал при импульсе $k$

$$
w_{c}(k)=|c(k)|^{2}, \quad w_{d}(k)=|d(k)|^{2} .
$$

Приведем схему решения задачи. Пусть нам известны решения уравнения Шредингера во всех трех областях, показанных на рис. 2. Пусть $\psi_{+}(x)$ и $\psi_{-}(x)$ являются решениями уравнения Шредингера в области I с нужными асимптотиками. Тогда

$$
\begin{gathered}
\Psi_{\mathrm{I}}(x)=\psi_{+}(x)-c \psi_{-}(x), \\
\psi_{+}(x) \rightarrow e^{i k x / \hbar}, \quad x \rightarrow-\infty . \\
\psi_{-}(x) \rightarrow e^{-i k x / \hbar},
\end{gathered}
$$

Удобно ввести обозначения

$$
\begin{gathered}
V_{\mathrm{I}}(x)=V_{+}(x)-c V_{-}(x), \\
V_{\mathrm{I}}(x)=\left(\begin{array}{c}
\Psi_{\mathrm{I}}(x) \\
\Psi_{\mathrm{I}}^{\prime}(x)
\end{array}\right), \quad V_{+}(x)=\left(\begin{array}{c}
\psi_{+}(x) \\
\psi_{+}^{\prime}(x)
\end{array}\right), \quad V_{-}(x)=\left(\begin{array}{c}
\psi_{-}(x) \\
\psi_{-}^{\prime}(x)
\end{array}\right) .
\end{gathered}
$$

Пусть $\phi_{+}(x)$ и $\phi_{-}(x)$ являются ненормированными решениями уравнения Шредингера в области II, причем функция $\phi_{+}(x)$ растет, а функция $\phi_{-}(x)$ убывает с ростом $x$. Тогда

$$
\Psi_{\mathrm{II}}(x)=B_{1} \phi_{+}(x)+B_{2} \phi_{-}(x),
$$


где $B_{1}$ и $B_{2}$ - некоторые постоянные. Удобно ввести представление

$$
\begin{gathered}
V_{\mathrm{II}}(x)=\left(\begin{array}{l}
\Psi_{\mathrm{II}}(x) \\
\Psi_{\mathrm{II}}^{\prime}(x)
\end{array}\right)=\left(\begin{array}{ll}
\phi_{+}(x) & \phi_{-}(x) \\
\phi_{+}^{\prime}(x) & \phi_{-}^{\prime}(x)
\end{array}\right)\left(\begin{array}{l}
B_{1} \\
B_{2}
\end{array}\right)=D(x) B, \\
D(x)=\left(\begin{array}{ll}
\phi_{+}(x) & \phi_{-}(x) \\
\phi_{+}^{\prime}(x) & \phi_{-}^{\prime}(x)
\end{array}\right), \quad B=\left(\begin{array}{l}
B_{1} \\
B_{2}
\end{array}\right) .
\end{gathered}
$$

Пусть $\mathcal{W}$ - вронскиан этих двух независимых решений:

$$
\mathcal{W}=\phi_{+}(x) \phi_{-}^{\prime}(x)-\phi_{+}^{\prime}(x) \phi_{-}(x)=\text { const } .
$$

В области III имеется только одно решение уравнения Шредингера, имеющее в асимптотике расходящуюся волну:

$$
\Psi_{\text {III }}(x)=d \chi(x), \quad \chi(x) \rightarrow e^{i k x / \hbar}, \quad x \rightarrow \infty .
$$

Введем обозначение

$$
V_{\mathrm{III}}(x)=\left(\begin{array}{c}
\Psi_{\mathrm{III}}(x) \\
\Psi_{\mathrm{III}}^{\prime}(x)
\end{array}\right)=d V_{\text {out }}(x), \quad V_{\text {out }}(x)=\left(\begin{array}{c}
\chi(x) \\
\chi^{\prime}(x)
\end{array}\right) .
$$

Полученные решения должны быть сшиты на границах областей I и II, а также II и III. Условия сшивки решений имеют вид

$$
V_{\mathrm{I}}(a)=V_{\mathrm{II}}(a), \quad V_{\mathrm{II}}(b)=V_{\mathrm{III}}(b)
$$

или

$$
V_{+}(a)-c V_{-}(a)=D(a) B, \quad D(b) B=d V_{\text {out }}(b) .
$$

Исключим постоянные $B$, получим

$$
V_{+}(a)-c V_{-}(a)=d D(a) D^{-1}(b) V_{\text {out }}(b)
$$

Для $D(a)$ и $D^{-1}(b)$ имеем

$$
D(a)=\left(\begin{array}{ll}
\phi_{+}(a) & \phi_{-}(a) \\
\phi_{+}^{\prime}(a) & \phi_{-}^{\prime}(a)
\end{array}\right), \quad D^{-1}(b)=\frac{1}{\mathcal{W}}\left(\begin{array}{cc}
\phi_{-}^{\prime}(b) & -\phi_{-}(b) \\
-\phi_{+}^{\prime}(b) & \phi_{+}(b)
\end{array}\right)
$$

В предположении, что барьер достаточно велик, т. е. при $b \gg a$ выполнены условия $\left|\phi_{+}(b)\right| \gg\left|\phi_{+}(a)\right|$ и $\left|\phi_{-}(a)\right| \gg\left|\phi_{-}(b)\right|$, получим

$$
D(a) D^{-1}(b) \Rightarrow-\frac{\phi_{+}(b) \phi_{-}(a)}{\mathcal{W}}\left(\begin{array}{cc}
1 \\
-\frac{\phi_{-}^{\prime}(a)}{\phi_{-}(a)}
\end{array}\right)\left(\frac{\phi_{+}^{\prime}(b)}{\phi_{+}(b)}-1\right) .
$$

Из равенства (49) надо исключить параметр с. Для этого введем антисимметричную матрицу $\epsilon$, так что

$$
\left(V_{-}(a) \epsilon V_{-}(a)\right)=0, \quad \epsilon=\left(\begin{array}{cc}
0 & 1 \\
-1 & 0
\end{array}\right)
$$


Отсюда получаем

$$
\left(V_{-}(a) \epsilon V_{+}(a)\right)=d\left(V_{-}(a) \epsilon D(a) D^{-1}(b) V_{\text {out }}(b)\right),
$$

причем

$$
\left(V_{-}(a) \epsilon V_{+}(a)\right)=\psi_{-}(a) \psi_{+}^{\prime}(a)-\psi_{-}^{\prime}(a) \psi_{+}(a)=i \frac{2 k}{\hbar} .
$$

Окончательно вероятность прохождения сквозь потенциальный барьер равна

$$
w=|d|^{2}=\left|\frac{\left(V_{-}(a) \epsilon V_{+}(a)\right)}{\left(V_{-}(a) T(a, b) V_{\text {out }}(b)\right)}\right|^{2}=\frac{4 k^{2} / \hbar^{2}}{\left|\left(V_{-}(a) T(a, b) V_{\text {out }}(b)\right)\right|^{2}},
$$

где матрица $T(a, b)$ определена как

$$
T(a, b)=\epsilon D(a) D^{-1}(b)=\frac{\phi_{+}(b) \phi_{-}(a)}{\mathcal{W}}\left(\begin{array}{c}
\frac{\phi_{-}^{\prime}(a)}{\phi_{-}(a)} \\
1
\end{array}\right)\left(\frac{\phi_{+}^{\prime}(b)}{\phi_{+}(b)}-1\right) .
$$

Воспользовавшись полученными выше представлениями, получим

$$
\begin{aligned}
& \left(V_{-}(a) T(a, b) V_{\text {out }}(b)\right)= \\
& \quad=\frac{\phi_{+}(b) \phi_{-}(a)}{\mathcal{W}} \psi_{-}(a) \chi(b)\left(\frac{\phi_{-}^{\prime}(a)}{\phi_{-}(a)}+\frac{\psi_{-}^{\prime}(a)}{\psi_{-}(a)}\right)\left(\frac{\phi_{+}^{\prime}(b)}{\phi_{+}(b)}-\frac{\chi^{\prime}(b)}{\chi(b)}\right) .
\end{aligned}
$$

7.1. Квазиклассическое приближение. Получим вероятность прохождения сквозь потенциальный барьер в квазиклассическом приближении. Волновые функции определены формулами (40) и (41).

В области I функции, удовлетворяющие краевым условиям (43), записываются каK

$$
V_{-}(x)=\sqrt{\frac{k}{P(x)}} \exp \left[\mp \frac{i}{\hbar} \int_{x}^{a} d x^{\prime} P\left(x^{\prime}\right)\right]\left( \pm \frac{i}{\hbar} P(x)-\frac{P^{\prime}(x)}{2 P(x)}\right) .
$$

В точке поворота $x=a$

$$
V_{-}(a)=\sqrt{\frac{k}{\kappa_{a}}}\left( \pm \frac{i}{\hbar} \kappa_{a}+\frac{m V^{\prime}(a)}{2 \kappa_{a}^{2}}\right)
$$

В области II имеем

$$
\phi_{ \pm}(x)=\frac{1}{\sqrt{|P(x)|}} \exp \left[ \pm \frac{1}{\hbar} \int_{a}^{x} d x^{\prime}\left|P\left(x^{\prime}\right)\right|\right]
$$

и

$$
\mathcal{W}=\phi_{+}(x) \phi_{-}^{\prime}(x)-\phi_{+}^{\prime}(x) \phi_{-}(x)=-\frac{2}{\hbar} .
$$

Матрица $T(a, b)$ равна

$$
\begin{aligned}
& T(a, b)=\epsilon D(a) D^{-1}(b)= \\
& =\frac{\hbar}{2 \sqrt{\kappa_{a} \kappa_{b}}} \exp \left[\frac{1}{\hbar} \int_{a}^{b} d x^{\prime}\left|P\left(x^{\prime}\right)\right|\right]\left(\begin{array}{c}
-\frac{\kappa_{a}}{\hbar}-\frac{m V^{\prime}(a)}{2 \kappa_{a}^{2}} \\
1
\end{array}\right)\left(-\frac{\kappa_{b}}{\hbar}+\frac{m V^{\prime}(b)}{2 \kappa_{b}} 1\right) .
\end{aligned}
$$


В области III имеем

$$
V_{\text {out }}(b)=d \sqrt{\frac{k}{\kappa_{b}}}\left(i \frac{\kappa_{b}}{\hbar}-\frac{m V^{\prime}(b)}{2 \kappa_{b}^{2}}\right) .
$$

Подставим квазиклассические функции в формулу (51), получим

$$
\begin{gathered}
\left(V_{+}(a) \epsilon V_{-}(a)\right)=-\frac{2 i k}{\hbar} \\
\left(V_{-}(a) T(a, b) V_{\text {out }}(b)\right)=-\exp \left[\frac{1}{\hbar} \int_{a}^{b} d x^{\prime}\left|P\left(x^{\prime}\right)\right|\right] d \frac{k}{\hbar}\left(\frac{-1+i}{\sqrt{2}}\right)^{2} .
\end{gathered}
$$

Таким образом, вероятность прохождения сквозь потенциальный барьер в квазиклассическом приближении равна

$$
w=|d|^{2}=\left|\frac{\left(V_{+}(a) \epsilon V_{-}(a)\right)}{\left(V_{-}(a) T(a, b) V_{\text {out }}(b)\right)}\right|^{2}=4\left|\exp \left[-\frac{1}{\hbar} \int_{a}^{b} d x^{\prime} \sqrt{-P^{2}\left(x^{\prime}\right)}\right]\right|^{2},
$$

где

$$
P^{2}(x)=k^{2}-2 m V(x)+\kappa^{2}(x), \quad \kappa(x)=e^{-\pi i / 6}\left(\frac{m V^{\prime}(x) \hbar}{2}\right)^{1 / 3} .
$$

Полученное выражение отличается на коэффициент 4 от стандартного выражения, приводимого в учебной литературе (см., например, [9]). Дело в том, что обычно пренебрегается функцией, растущей в нефизической области, однако именно она дает главный вклад в коэффициент прохождения.

\section{8. УПРУГОЕ РАССЕЯНИЕ}

Имеется ряд исследований (см., например, [13]-[16] и последнюю работу [17], где приведены ссылки на предыдущие статьи), в которых метод функционального интегрирования применялся к теории потенциального рассеяния в нерелятивистской квантовой механике с целью получения представления для амплитуды рассеяния в форме интеграла по путям. Основная схема рассуждений состояла в том, чтобы исходя из временно́го уравнения Шредингера написать представление для $S$-матрицы как амплитуды перехода от состояний при $t=-\infty$ к состояниям при $t=\infty$, а затем выделить $T$-матрицу, из которой, в свою очередь, надо выделить $\delta$-функцию, отвечающую за сохранение энергии в начальном и конечном состояниях. Мы не будем критиковать эти работы, но отметим, что искомое представление может быть непосредственно получено из стационарного уравнения Шредингера.

Задача рассеяния ставится следующим образом. Предполагается, что потенциал $V(r)$ достаточно быстро убывает на больших расстояниях. Надо найти решение стационарного уравнения Шредингера (18) для непрерывного спектра при $E=k^{2} / 2 m$, удовлетворяющего асимптотическим граничным условиям

$$
\Psi(\mathbf{x}) \longrightarrow e^{i \mathbf{k x}}+f(k, \theta) \frac{e^{i k r}}{r}, \quad r \rightarrow \infty
$$

где $f(k, \theta)$ - искомая амплитуда рассеяния, которую необходимо вычислить. 
Будем искать решение в форме

$$
\Psi(\mathbf{x})=e^{i \mathbf{k x}}+\Phi(\mathbf{x}) .
$$

Функция $\Phi(\mathbf{x})$ удовлетворяет уравнению

$$
\left(-\frac{1}{2 m} \frac{d^{2}}{d \mathbf{x}^{2}}+V(\mathbf{x})-\frac{\mathbf{k}^{2}}{2 m}\right) \Phi(\mathbf{x})=-V(\mathbf{x}) e^{i \mathbf{k x}},
$$

решение которого можно записать как

$$
\Phi(\mathbf{x})=-\frac{1}{-\frac{1}{2 m} \frac{d^{2}}{d \mathbf{x}^{2}}+V(\mathbf{x})-\frac{\mathbf{k}^{2}}{2 m}-i 0} V(\mathbf{x}) e^{i \mathbf{k x}} .
$$

Представим это решение в виде функционального интеграла, проводя стандартные преобразования:

$$
\begin{aligned}
\Phi(\mathbf{x})= & -i \int_{0}^{\infty} d t \exp \left\{-i t\left[-\frac{1}{2 m} \frac{d^{2}}{d \mathbf{x}^{2}}-\frac{\mathbf{k}^{2}}{2 m}+V(\mathbf{x})-i 0\right]\right\} V(\mathbf{x}) e^{i \mathbf{k x}}= \\
= & -i \int_{0}^{\infty} \frac{m^{3 / 2} d t}{(2 \pi i t)^{3 / 2}} e^{i t \mathbf{k}^{2} / 2 m} \int d \mathbf{y} e^{i \mathbf{y}^{2} m / 2 t} \int \frac{D \boldsymbol{\xi}}{C} \times \\
& \times \exp \left\{i \int_{0}^{t} d \tau\left[\frac{m}{2} \dot{\boldsymbol{\xi}}^{2}(\tau)-V\left(\mathbf{x}+\left(1-\frac{\tau}{t}\right) \mathbf{y}-\boldsymbol{\xi}(\tau)\right)\right]\right\} \times \\
& \times V(\mathbf{x}+\mathbf{y}) e^{i \mathbf{k}(\mathbf{x}+\mathbf{y})}=-i \int d \mathbf{y} V(\mathbf{y}) e^{i \mathbf{k y}} I(\mathbf{y}, \mathbf{x}, \mathbf{k}),
\end{aligned}
$$

где

$$
\begin{aligned}
I(\mathbf{y}, \mathbf{x}, \mathbf{k})= & \int_{0}^{\infty} \frac{m^{3 / 2} d t}{(2 \pi i t)^{3 / 2}} \exp \left[\frac{i}{2}\left(\frac{t \mathbf{k}^{2}}{m}+\frac{(\mathbf{x}-\mathbf{y})^{2} m}{t}\right)\right] \int \frac{D \boldsymbol{\xi}}{C} \times \\
& \times \exp \left\{i \int_{0}^{t} d \tau\left[\frac{m}{2} \dot{\boldsymbol{\xi}}^{2}(\tau)-V\left(\frac{\mathbf{x}}{t} \tau+\left(1-\frac{\tau}{t}\right) \mathbf{y}-\boldsymbol{\xi}\left(\tau^{\prime}\right)\right)\right]\right\}
\end{aligned}
$$

с краевыми условиями $\boldsymbol{\xi}(0)=\boldsymbol{\xi}(t)=0$. Нас интересует поведение функции $I(\mathbf{y}, \mathbf{x}, \mathbf{k})$ в пределе $|\mathbf{x}|=r \rightarrow \infty$. Сделаем в интеграле (61) замену переменных $t=r s$, положим $\mathbf{n}=\mathbf{x} / r$, тогда при больших $r$ получим

$$
\begin{aligned}
I(\mathbf{y}, \mathbf{x}, \mathbf{k})= & \frac{m^{3 / 2}}{\sqrt{r}} \int_{0}^{\infty} \frac{d s}{(2 \pi i s)^{3 / 2}} \exp \left\{i \frac{r}{2}\left[\frac{s \mathbf{k}^{2}}{m}+\frac{(\mathbf{n}-\mathbf{y} / r)^{2} m}{s}\right]\right\} \int \frac{D \boldsymbol{\xi}}{C} \times \\
& \times \exp \left\{\frac{i}{2} \int_{0}^{r s} d \tau\left[\dot{\boldsymbol{\xi}}^{2}(\tau)+W\left(\frac{\mathbf{n}}{s}+\left(1-\frac{\tau}{r s}\right) \mathbf{y}-\boldsymbol{\xi}\left(\tau^{\prime}\right)\right)\right]\right\} \rightarrow \\
\rightarrow & \frac{m^{3 / 2}}{\sqrt{r}} \int_{0}^{\infty} \frac{d s}{(2 \pi i s)^{3 / 2}} \exp \left[i \frac{r}{2}\left(\frac{s \mathbf{k}^{2}}{m}+\frac{m}{s}\right)-i \frac{\mathbf{n y} m}{s}\right] \int \frac{D \boldsymbol{\xi}}{C} \times \\
& \times \exp \left\{i \int_{0}^{\infty} d \tau\left[\frac{m}{2} \dot{\boldsymbol{\xi}}^{2}(\tau)-V\left(\frac{\mathbf{n}}{s}+\mathbf{y}-\boldsymbol{\xi}(\tau)\right)\right]\right\} .
\end{aligned}
$$


При больших $r$ интеграл по $s$ вычисляется методом перевала, результат имеет вид

$$
I(\mathbf{y}, \mathbf{x}, \mathbf{k}) \rightarrow \frac{m e^{i k r}}{2 \pi i r} e^{-i k \mathbf{n y}} \int \frac{D \boldsymbol{\xi}}{C} \exp \left\{i \int_{0}^{\infty} d \tau\left[\frac{m}{2} \dot{\boldsymbol{\xi}}^{2}(\tau)-V\left(\frac{\mathbf{n} k}{m} \tau+\mathbf{y}-\boldsymbol{\xi}(\tau)\right)\right]\right\}
$$

Таким образом, для амплитуды упругого рассеяния получаем представление

$$
f(k, \theta)=\frac{m}{2 \pi} \int d \mathbf{y} V(\mathbf{y}) e^{i \mathbf{q y}} \int \frac{D \boldsymbol{\xi}}{C} \exp \left\{i \int_{0}^{\infty} d \tau\left[\frac{m}{2} \dot{\boldsymbol{\xi}}^{2}(\tau)-V\left(\mathbf{v}_{\text {out }} \tau+\mathbf{y}-\boldsymbol{\xi}(\tau)\right)\right]\right\}
$$

с краевыми условиями $\boldsymbol{\xi}(0)=\boldsymbol{\xi}(\infty)=0$ и

$$
\mathbf{q}=\mathbf{k}_{\text {in }}-\mathbf{k}_{\text {out }}, \quad \mathbf{v}_{\text {out }}=\frac{\mathbf{k}_{\text {out }}}{m}=\frac{k \mathbf{n}}{m}, \quad \mathbf{q}^{2}=4 k^{2} \sin ^{2} \frac{\theta}{2} .
$$

\section{9. ЗАКЛЮЧЕНИЕ}

Получено представление решений однородных уравнений второго порядка в форме функционального интеграла, или интеграла по путям. Полученное представление амплитуды рассеяния может быть использовано при решении задачи рассеяния на сложных объектах: молекулах, связанных состояниях и т. п. При этом могут быть использованы известные методы вычисления функциональных интегралов: теория возмущений, квазиклассическое приближение, вариационные оценки и гауссово эквивалентное представление (см., например, [7], [8]).

\section{Список литературы}

[1] Р. Фейнман, А. Хибс, Квантовая механика и интеграль по траекториям, Мир, М., 1968.

[2] H. Kleinert, Path Integrals in Quantum Mechanics, Statistics, Polymer Physics and Financial Marketing, World Scientific, Hackensack, NJ, 2006.

[3] В. Н. Попов, Континуальные интегралы в квантовой теории поля и статистической механике, Атомиздат, М., 1976.

[4] Ж. Зинн-Жюстин, Континуалъный интеграл в квантовой механике, Физматлит, М., 2006.

[5] A. Das, Field Theory. A Path Integral Approach, World Scientific Lecture Notes in Physics, 75, World Scientific, New York, 2006.

[6] E. Witten, "A new look at the path integral of quantum mechanics", Perspectives in Mathematics and Physics, Surveys in Differential Geometry, 15, eds. T. Mrowka et al., Internat. Press, Somerville, MA, 2011, 345-419, arXiv: 1009.6032.

[7] V. Dineykhan, G. V. Efimov, G. Ganbold, S. N. Nedelko, Oscillator Representation in Quantun Physics, v. 26, Lecture Notes in Physics. New Series m, Springer, Berlin, 1995.

[8] Г. В. Ефимов, Метод функиионального интегрирования, Международный университет природы, общества и человека "Дубна", Дубна, 2008.

[9] Л. Д. Ландау, Е. М. Лифшиц, Курс теоретической физики, т. 3: Квантовая механика. Нерелятивистская теория, Наука, М., 1988.

[10] Г. Сеге, Ортогональные многочлены, Физматгиз, М., 1962.

[11] I. M. Gel'fand, A. M. Yaglom, J. Math. Phys., 1:1 (1960), 48-69.

[12] J. Ankerhold, Quantum Tunneling in Complex Systems. The Semiclassical Approach, Springer Tracts in Modern Physics, 224, Springer, Berlin, 2007. 
[13] D. Gelman, L. Spruch, J. Math. Phys., 10:12 (1969), 2240-2254.

[14] W.B. Campbell, P. Finkler, C.E. Jones, M. N. Misheloff, Phys. Rev. D, 12:8 (1975), 2363-2369.

[15] А. В. Васильев, А. В. Кузьменко, ТМФ, 31:3 (1977), 313-326.

[16] Б. М. Барбашов, В. В. Нестеренко, Приближение эйконала для процессов высокоэнергетического рассеяния частии, Изд-во МГУ, М., 1977.

[17] J. Carron, R. Rosenfelder, Eur. Phys. J. A, 45:2 (2010), 193-215.

Поступила в редакцию 22.06.2011, после доработки 3.10 .2011 